analysed by ANOVA test. The study was approved by the Hospital Ethical Committee.

Results Among all the women enrolled, 25 were considered healthy, 18 received a diagnosis of $\mathrm{BV}$ and 22 were positive for CT. PCA revealed that the vaginal microbiome of healthy and BV-subjects were clearly distinct and that CT-positive women were more similar to healthy women rather than to BV-positives, both for microbial composition and for metabolic profile. The mean GV DNA load was significantly different between the groups $(p=0.03)$ : healthy and CT positive women showed similar and lower mean loads compared to BV group. At a metabolic level, significantly higher concentrations of formate, ethanolamine and methylamine were found in BVpatients, while tryptophan and lactate were more present in healthy and CT-positive women.

Conclusion Specific microbial and metabolic signatures characterise different clinical conditions of the vaginal tract. In this context, CT-positive women are definitely more similar to healthy than BV-subjects.

\section{P1.14 EVALUATION OF THE APTIMA ASSAYS FOR THE DETECTION OF BACTERIAL SEXUALLY TRANSMITTED INFECTIONS IN A SELECTED POPULATION OF WOMEN}

${ }^{1}$ Claudio Foschi, ${ }^{2}$ Nicoletta Banzola, ${ }^{2}$ Valeria Gaspari, ${ }^{2}$ Antonietta D'antuono, ${ }^{1}$ Roberto Cevenini, 'Antonella Marangoni. 'Microbiology Dimes; University of Bologna, Bologna - Italy; ${ }^{2}$ Dermatology, Dimes, University of Bologna, Bologna - Italy

10.1136/sextrans-2017-053264.122

Introduction: Chlamydia trachomatis (CT), Neisseria gonorrhoeae (NG) and Mycoplasma genitalium (MG) represent the most common agents of bacterial sexually transmitted infections (STIs), worldwide. In women, uro-genital infections caused by these microorganisms are often asymptomatic and, left untreated, can lead to several sequelae. Nucleic acid amplification techniques (NAATs) have become the reference methods for the diagnosis, thanks to the suitability for different specimens and the outstanding sensitivity and specificity. The aim of this study was to assess the performance of Aptima Assays for CT, NG and MG detection in a group of selected women, by a head-to-head comparison with other NAATs. Moreover, an evaluation about the suitability for the Aptima assays with one of the most used swab collection device (E-Swab; Copan) was carried out.

Methods Routinely, all the women attending the STI Outpatients Clinic of Sant'Orsola-Malpighi Hospital of Bologna (Italy) complaining of genital STI-related symptoms or reporting unsafe intercourse, are managed as follows. After a clinical visit, a sample of first-void urines and a vaginal swab collected in E-swab, are obtained for CT, NG and MG detection. A duplex real-time PCR (Versant CT/GC DNA 1.0 assay; Siemens) is used for CT and NG detection, while, MG presence is investigated by a home-made PCR, starting from the remaining eluate of Versant PCR plate. From January 2016, a total of 100 patients were selected and their samples were also tested with Aptima assays. Previously frozen samples were thawed and transferred to the suitable collection devices for Aptima assays: in particular, $2 \mathrm{ml}$ of urines and $100 \mu \mathrm{l}$ of vaginal E-swab were used. All the specimens were processed by Aptima Combo $2^{\circledast}$ for CT and NG detection and by the Aptima $^{\circledast 4}$ Mycoplasma genitalium assay for $\mathrm{MG}$ infection diagnosis. These assays were run on Panther system (Hologic). A comparison between the different molecular methods, stratified by type of sample and microorganism, was conducted.

Results In the group of 100 women selected, 25 patients were positive for CT, 4 for NG and 6 for MG. One case of CTNG and two cases of CT-MG co-infections were found. Interestingly, more than 50\% of CT-positive women were completely asymptomatic. By the routine tests, all positive cases were simultaneously found both on the urine sample and on the vaginal swab, except for $3 \mathrm{CT}, 1 \mathrm{NG}$ and $1 \mathrm{MG}$ infections, detected only on the vaginal swab. A complete concordance with Aptima assays, both for the type of sample and microorganism was found, with only one discordant result (a CT case detected by Versant on urines and vaginal swab, found by Aptima only on urines). Any interference due to the different liquid components of E-Swab was excluded.

Conclusion Given the outstanding performance, Aptima assays can represent an excellent choice for CT, NG and MG molecular detection. Moreover, it is noteworthy that Aptima assays allow testing of specimens collected by E-Swab, enabling the possibility to use the same sample for both NG molecular detection and culture.

\section{P1.15 TRICHOMONAS VAGINALIS IN HUMAN IMMUNODEFICIENCY VIRUS-INFECTED PREGNANT WOMEN: PREVALENCE, DETECTION, AND APPLICATION OF PCR CYCLE-THRESHOLD VALUES}

${ }^{1}$ Collin Price, ${ }^{2}$ Davvie Olivier, ${ }^{2}$ Lindsey De Vos, ${ }^{2}$ Phuti Ngwepe, ${ }^{2}$ Maanda Mudau, ${ }^{3}$ Janré Steyn, ${ }^{2}$ Andrew Biundo, ${ }^{3}$ Remco Ph Peters, ${ }^{3}$ Marleen M Kock, ${ }^{2}$ Andrew MedinaMarino, 'Jeffrey D Klausner. 'Department of Infectious Diseases, David Geffen School of Medicine, Ucla, Los Angeles, USA; ${ }^{2}$ Foundation for Professional Development, Pretoria, South African Republic; ${ }^{3}$ Department of Medical Microbiology, University of Pretoria, Pretoria, South African Republic

\subsection{6/sextrans-2017-053264.123}

Introduction: Trichomonas vaginalis (TV) is a sexually transmitted infection (STI) associated with increased transmission of human immunodeficiency virus (HIV) and significant adverse birth outcomes. Although culture is often used in the diagnosis of TV, molecular diagnosis is rapid, accurate, and data-rich.

Methods Women were recruited from two clinics in South Africa as part of a study assessing point-of-care polymerase chain reaction (PCR) diagnosis and treatment of three STIs. HIV-infected pregnant women were screened for TV using the Xpert TV (Cepheid, Sunnyvale, CA). Each woman who tested TV PCR-positive provided an additional sample for culture (InPouch TV, Biomed, San Jose, CA). We compared TV detection between PCR and culture, and used non-parametric statistics to compare cycle threshold $(\mathrm{Ct})$ values among culture results and treatment outcomes.

Results By December 31 $1^{\text {st }}, 2016,200$ women were enrolled and $52(26 \%)$ tested TV PCR-positive. Baseline cultures were obtained from 41 (79\%) of the TV PCR-positive women, and 22 (54\%) were culture-positive. The median baseline $\mathrm{Ct}$ of the TV PCR-positive/culture-positive group was 24.0 (IQR: 5.1) vs. 38.0 (IQR: 3.8) among those TV PCR-positive/culture-negative $(\mathrm{p}<0.05)$. Forty-two women returned for a 3 week test-of-cure (ToC), and 10 (24\%) were still TV PCRpositive. Of the women who remained TV PCR-positive at 
ToC, the median baseline Ct was 26.9 (IQR: 12.5) vs. 29.3 (IQR: 14.8) among those TV PCR-negative at ToC $(\mathrm{p}=0.52)$. Among 7 women who remained TV PCR-positive, the median baseline Ct was 26.4 (IQR: 6.7) vs. 26.2 (IQR: 6.7) at $\mathrm{ToC}$ $(\mathrm{p}>0.05)$.

Conclusion The prevalence of TV in our sample of South African HIV-infected pregnant women was similar to prior studies. At baseline, culture detected only half of the cases that were positive by TV PCR. The culture-negative cases had significantly higher $\mathrm{Ct}$ values, indicating a lower burden of TV nucleic acid. Baseline $\mathrm{Ct}$ values did not predict response to TV treatment. Among women testing persistently TV PCRpositive, Ct values did not change between baseline and ToC.

\section{P1.16 ACQUISITION OF AZITHROMYCIN RESISTANCE IN NEISSERIA GONORRHOEAE VIA INTRAGENUS RECOMBINATION}

Crista Wadsworth, Yonatan Grad. Harvard Th Chan School of Public Health, Boston, MA, USA, USA

\subsection{6/sextrans-2017-053264.124}

Introduction Reduced susceptibility to azithromycin ( $\mathrm{Azi}^{\mathrm{RS}}$ ) is increasing in frequency in Neisseria gonorrhoeae. We have shown that non-gonococcal alleles at the mtr operon, encoding the MtrCDE efflux pump and the transcription repressor, are associated with $\mathrm{Azi}^{\mathrm{RS}}$ and sought to define further their role in resistance.

Methods We transformed a susceptible strain (MIC $<0.125 \mu \mathrm{g} / \mathrm{mL}$ ) using gDNA from gonococcal donors carrying mosaic $m t r$ loci and selected for $\mathrm{Azi}^{\mathrm{RS}}$ (MIC $\geq 2 \mu \mathrm{g}$ ) $\mathrm{mL}$ ) transformants. We sequenced the transformants' genomes to define the transformed DNA, compared growth rates of parent and transformant strains, described the phylogenetic distribution of $m t r R, m t r C, m t r D$, and $m t r E$ alleles across Neisserial species, and defined the sequence diversity and $\pi$ at these loci.

Results Transformation studies confirm mosaic $m t r$ alleles cause $\mathrm{Azi}^{\mathrm{RS}}$. Phylogenetic patterns support frequent $\mathrm{Azi}^{\mathrm{RS}}$-associated recombination of $m t r R, m t r C$, and $m t r D$ between $N$. gonorrhoeae and other Neisseria. Conversely, $16 S$ and $m t r E$ displayed almost exclusive species-based clade topology and no recombination events associated with $\mathrm{Azi}^{\mathrm{RS}}$. Within gonococcal populations, reduced nucleotide diversity at $m t r E \quad(n=1102$, $\pi=0.0054)$ compared to $m t r R C D(\pi=0.014)$ suggests either equal rates of interspecific recombination across the $m t r$ operon with increased levels of purifying selection acting to purge the introduction of novel alleles at $m t r E$, or a 'hotspot' of interspecific recombination at $m t r R C D$. Recombination of mosaic $m t r$ alleles into novel gonococcal genomic backgrounds does not deleteriously affect growth rate in vitro, raising questions regarding the associated fitness cost.

Conclusion This work supports that Neisseria serve as a reservoir of gonococcal Azi ${ }^{\mathrm{RS}}$ through interchange of $\mathrm{mtr}$ alleles. The genomic epidemiological evidence of multiple acquisitions of these alleles underscores the importance of screening for $m t r$ mosaics to prevent outbreaks of $\mathrm{Azi}^{\mathrm{RS}}$.

\section{P1.17 EFFECTIVENESS OF SYNDORMIC CASE MANAGEMENTS (SCM) IN CONTROL OF SEXUALLY TRANSMITTED INFECTION AND ANTIBIOTIC RESISTANCE}

${ }^{1}$ Subash C Sonkar, ${ }^{2} \mathrm{R}$ Bharti, ${ }^{1} \mathrm{~K}$ Wasnik, ${ }^{2} \mathrm{~A}$ Kumar, ${ }^{1}$ Mishra Pk, ${ }^{2} \mathrm{P}$ Mittal, ${ }^{1} \mathrm{D}$ Saluja. ${ }^{1}$ Medical Biotechnology Lab, Dr. B. R. Ambedkar Centre For Biomedical Research, University of Delhi, D, Delhi, India; ${ }^{2}$ Department of Obstetrics and Gynaecology, Vardhman Mahavir Medical College and Safdariung Hospital, New, New Delhi, India

\subsection{6/sextrans-2017-053264.125}

Introduction Utility of syndromic case management (SCM) in symptomatic women facing huge misdiagnosis/overtreatment problems due to lack of accurate and confirmed diagnosis. As a consequence antibiotic resistance has accelerated along with an increase in risk of infection to their partner.

Methods symptomatic women (18-56 years old) were recruited in the study and examined by clinician based on subjective judgment and treated vaginal discharge with various clinical symptoms causing microorganisms by NACO-NACP III Algorithms for STI/RTI and comparative analysis using PCRbased diagnostic assay.

Results We found that; 646/3200 (20.18\%) female patients reported vaginal discharge and recommended treatment for either CT, NG, TV and Candida and/or co-infection using pre-packed STI/RTI kits under NACP III. Based on PCR, 48/ $646(7.43 \%)$ subjects tested positive for infection with NG/ TV/CT. Amongst 46 patents, 28 (60.86\%) were correct and conformed diagnosis by PCR and SCM both. While 18/46 (39.14\%) were incomplete treated and overtreatment due to poor diagnosis. Out of 600/646 (92.87) treated patients were uninfected of these three pathogens. Based on PCR and SCM, prevalence of TV, NG and CT were shows huge variations. As a result, SCM is inaccurate as infection caused by any of these pathogens showed similar symptoms: vaginitis, cervicitis, genital ulcers, AVD and LAP. The recent increases of misdiagnosis, overtreatment and antibiotic resistance are cause for public health concern. Here our results clearly demonstrate that prevalence of CT and NG is still significant among female patients.

Conclusion The study underpins the need to implement diagnostic assays for identification of causative pathogen before implementing antibiotic treatment to patients with vaginal discharge. It also divulges the need to review the use of SCM for controlling sexually transmitted diseases.

\section{P1.18 EVALUATION OF THE COBAS ${ }^{\circledR}$ HSV 1 AND 2 TEST IN A JAPANESE POPULATION}

${ }^{1}$ Elizabeth Marlowe, ${ }^{2}$ Takashi Kawana, ${ }^{3}$ Tomohiko Onoe, ${ }^{4}$ Yasuhiko Onoe, ${ }^{5}$ Masayuki Sawamura, ${ }^{6}$ Kenichi Hayakawa, ${ }^{1}$ Michael Lewinski, ${ }^{7}$ Rui Ohse, ${ }^{7}$ Ai Takiyama. ${ }^{1}$ Roche Molecular Systems, Pleasanton, USA; ${ }^{2}$ Teikyo University Mizonokuchi Hospital, Kanagawa - Japan; ${ }^{3}$ The Jikei University Katsushika Medical Centre, Tokyo - Japan; ${ }^{4}$ Miyamoto-Cho Central Clinic, Kanagawa - Japan; ${ }^{5}$ Shinjuku Sakura Clinic, Tokyo - Japan; ${ }^{6}$ Seiwakai Hayakawa Clinic Medical Corporation, Osaka - Japan; ${ }^{7}$ Roche Diagnostics K.K., Tokyo - Japan

\subsection{6/sextrans-2017-053264.126}

Introduction Herpes is a common cause of sexually transmitted disease, which is often asymptomatic. Identification of genital herpes can impact the clinical management of patients who are HIV positive, immunosuppressed, pregnant, and individuals with HSV seronegative partners. The objective of this 